\title{
Disconnected glass-glass transitions and swallowtail bifurcations in microscopic spin models with facilitated dynamics
}

\author{
Mauro Sellitto \\ Dipartimento di Ingegneria Industriale e dell'Informazione, \\ Seconda Università di Napoli, Real Casa dell'Annunziata, I-81031 Aversa (CE), Italy
}

\begin{abstract}
It has been recently established that heterogeneous bootstrap percolation and related dynamic facilitation models exhibit a complex hierarchy of continuous and discontinuous transitions depending on lattice connectivity and kinetic constraints. Here the range of the previously observed phase diagram topologies and higher-order singularities is extended to disconnected glass-glass transitions and to cusp and swallowtail bifurcations (which can be generic and degenerate). The phase diagram and the order parameter for two different types of spin mixtures are analytically determined and an experimental realization of the new predictions emerging in our approach is suggested.
\end{abstract}




\section{INTRODUCTION}

Soft matter enjoys a rich variety of multiphase equilibria due to the subtle interplay of energetic and entropic forces acting on different length scales. When one of the parameters controlling the system thermodynamics is suddenly changed, however, the phase formation is generally hindered for kinetic reasons and one observes amorphous states with distinct physical (as opposed to chemical) features. Such novel states of structural arrest and the glass-to-glass transition they can possibly undergo, were first predicted within schematic Mode-Coupling Theory (MCT) [1], and have been subsequently observed in short-range attractive colloids, dense copolymer micellar solutions, and several model systems [2 15]. Their characterization is not only technologically important for material design but is also a theoretical challege, as there is no obvious way to discriminate, on a macroscopic observation scale, disordered patterns that are apparently featureless from a geometric standpoint.

Multiple glass states of short-range attractive colloids have been generally ascribed to structural changes of the cage that confines particle motion. For weak attraction the glass formation is driven by the usual steric effects at high packing density, while for strong attraction the tight clustering of particles leads to an amorphous state with a rigid gel-like structure which can exist even at very low packing density. When the control parameters (temperature and packing density) are continously changed, the transition between the two glass states can be either smooth or discontinuous. In the latter case the Debye-Waller factor undergoes an extra jump. Interestingly, it has been recently found that interparticle attraction is not an essential ingredient for the existence of multiple glasses. In fact, additional peculiar glass states do exist also in purely repulsive particle systems, such as hard spheres interacting with a square-shoulder potential [11-13] and binary mixtures with a disparate sizes of their components [14]. In these systems the competition between two repulsive length scales leads to distinct glasses dominated by packing properties on different length scales. Moreover, in a certain range of the control parameters, the glass-glass transition line results completely disconnected from the liquid phase [11, 13].

In this paper I show that similar intriguing features generally exist in microscopic onlattice models with facilitated dynamics. A new prediction that emerges from our calculations is that the disconnected glass-glass transition can appear in systems with either a discontinuous or a continuous liquid-glass transition. The framework naturally suggests 
that the latter possibility should be realized in fluid mixtures confined in a disordered porous matrix. Our theoretical analysis, which is complementary to MCT, confirms that these features are controlled primarily by the interplay of crowding effects on different microscopic lenght scales (due, e.g., to particles of dissimilar size), and are therefore generally expected in multicomponents systems whenever competing packing effects are important.

\section{HETEROGENEOUS FACILITATION APPROACH}

In the past decades there has been a long lasting effort to identify valuable on-lattice models enabling a detailed analysis of the microscopic mechanism behind the glass transition. A promising approach in this direction is provided by the heterogeneous extension of bootstrap percolation and dynamic facilitation ideas [16-19]. In this framework, the coarse-grained structure of a system is represented by an assembly of mesoscopic cells. Typically, to every cell $i$ is assigned a binary spin variable, $s_{i}= \pm 1$, depending on whether the local density is higher or lower than its average value. In the simplest case, no energetic interaction among cells is assumed, $\mathcal{H}=-h \sum_{i} s_{i}$. The crucial assumption is that the temporal evolution of the system is dictated by a kinetic constraint: density fluctuations in the cell $i$ occur if and only if there is a certain number, say $f_{i}$, of nearby low-density cells. $f_{i}$ is the local facilitation (or threshold) parameter which mimics the local cage effect and takes on values in the range $0 \leq f_{i} \leq z$, where $z$ is the lattice connectivity. The facilitation probability distribution $\pi(f)$ reflects the coexistence of different length scales in the system due to the presence of more or less mobile molecules, or of polymers with small and large gyration radius. In facilitated spin mixtures the average strength of kinetic constraints can be tuned smoothly by changing the populations of spins with different $f_{i}$, and one can thus explore a variety of different situations. Interesting results are obtained when the facilitated dynamics is cooperative, i.e., when $f_{i} \geq 2$. It turns out that is important to further distinguish the latter situation in strong, $f_{i}=z-1$ or $f_{i}=z$, and moderate $z-2 \geq f_{i} \geq 2$, cooperative dynamics. Ex-

plicit calculations and detailed numerical simulations have shown that the basic results of schematic MCT are well reproduced within this framework [19 21]. Qualitatively, when the dynamics is strongly cooperative, i.e., when the fraction of spins with $f_{i}=z-1$ or $f_{i}=z$ is larger than that with $2 \leq f_{i} \leq z-2$, the liquid-glass transition is continuous (and thus the incipient cluster of frozen spins is fractal); while in the opposite case it is discontinuous 
(with a corresponding core having a compact structure). In the intermediate situation there is a crossover between the two transitions that can be either smooth [20] or abrupt [21]. In the latter case, the discontinuous transition extends deep inside the glass phase, thus generating an extra glass-glass transition [21]. As we are going to see in the remaining part of the paper, unusual features occur when a more subtle competition between clusters of frozen spins with different facilitations is present in the spin mixture.

\section{FORMALISM}

Exploiting the analogy with heterogeneous bootstrap percolation on locally tree-like random graphs [18-20] one can see that the probability $B$ that a cell is, or can be brought, in the lower density state by only rearranging the state of the nearby $z-1$ cells obeys a self-consistent polynomial equation $\mathcal{Q}(B)=0$ where

$$
\mathcal{Q}(B)=1-B-p \Psi_{z-1}^{f}(1-B)
$$

and we have defined the auxiliary function

$$
\Psi_{z}^{f}(X)=\left\langle\sum_{n=0}^{f-1}\left(\begin{array}{l}
z \\
n
\end{array}\right) X^{z-n}(1-X)^{n}\right\rangle .
$$

Here $p$ is the fraction of higher density cells in thermal equilibrium at temperature $T$, where $p^{-1}=1+\exp \left(-h / k_{\mathrm{B}} T\right)$ and the angular brackets, $\langle\cdots\rangle$, represents the average over the

probability distribution of kinetic constraints, $\pi(f)$. Quite generally, one finds that at high temperature every cell can always change state, $B=1$, while at low enough temperature there is a fraction of spins which is unable to change state, $B<1$, and the system is therefore a glass. The detailed topology of the phase diagram at low temperature depends on the coefficients of the polynomial $\mathcal{Q}(B)$ and can be rather intricate. Interestingly, the self-consistent equation $\mathcal{Q}(B)=0$ has a formal structure quite similar to that satisfied by the nonergodicity parameter in MCT [21]. Accordingly, one can immediately draw the conclusion that $\mathcal{Q}(B)$ exhibits the same hierarchy of bifurcations of schematic MCT, provided that the facilitated dynamics selects the maximum root of $\mathcal{Q}$ (as it happens with MCT dynamics). A rigorous proof of the latter statement is still lacking and one must rely, at the moment, on numerical evidences and physical consistency arguments to support this conjecture. We recall that an 
$\mathrm{A}_{\ell}$ bifurcation occurs when the maximum root of $\mathcal{Q}$ has a degeneracy $\ell \geq 2$ and

$$
\frac{d^{n} \mathcal{Q}}{d B^{n}}=0, \quad n=0, \ldots, \ell-1 ; \quad \frac{d^{\ell} \mathcal{Q}}{d B^{\ell}} \neq 0
$$

The Taylor expansion of $\mathcal{Q}$ near the critical surface and Eqs. (3), immediately implies that the scaling form of the order parameter near an $\mathrm{A}_{\ell}$ bifurcation goes like $\epsilon^{1 / \ell}$, where $\epsilon$ is the distance from the critical surface (e.g., $\epsilon=T-T_{\mathrm{c}}$ ). Singularities of type $\mathrm{A}_{\ell}$ can be further distinguished in generic and degenerate depending on whether the order parameter, $\Phi$, changes abruptly or smoothly near the transition. To denote this latter case, we shall use the notation $A_{\ell}^{*}$. Near a degenerate singularity of type $A_{\ell}^{*}$ the order parameter behaves as $\Phi \sim \epsilon^{1 /(\ell-1)}$. In the original MCT literature the distinction between degenerate and generic singularities has been addressed only for the simple case $\ell=2$ (where such singularities were named type-A and type-B glass transitions) and $\ell=3$. As we shall see, such a distinction seems to apply well also for the more general case of higher-order singularities with $\ell>3$.

In the following, we shall focus on ternary mixtures with facilitation distribution

$$
\pi\left(f_{i}\right)=(1-q) \delta_{f_{i}, a}+(q-r) \delta_{f_{i}, b}+r \delta_{f_{i}, c}
$$

For such ternary mixtures, denoted here with $(a, b, c)$, we shall consider two distinct situations corresponding to facilitation values which are more or less sparse. For each situation, we determine the phase diagram and compute the fraction of permanently frozen spins, $\Phi$, which represents the actual order parameter in this framework. It is directly related to $B$ through the general relation

$$
\Phi=p \Psi_{z}^{f}(B)+(1-p) \Psi_{z}^{f}\left(p \Psi_{z-1}^{f-1}(B)\right)
$$

For sake of simplicity we shall consider hereafter only random graphs with fixed connectivity, i.e. Bethe lattices. Similar results are expected for more general random graphs with variable connectivity [19, 22, 23].

\section{MIXTURE $(2,3,4)$}

Let us first consider a mixture in which the facilitation values $(a, b, c)$ of spin populations are very close and $c=z-1$. The latter condition means that when the spin population with facilitation $f_{i}=c$ is large the dynamics becomes strongly cooperative. For our purposes the 


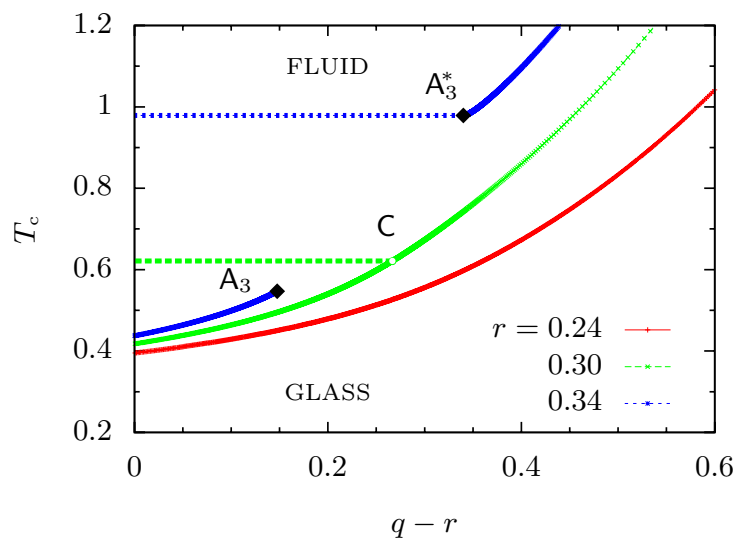

FIG. 1. A section of the phase diagram for the mixture $(2,3,4)$ on a Bethe lattice with connectivity $z=5$. Thick lines represent discontinuous liquid-glass and glass-glass transitions while thin dashed lines correspond to continuous liquid-glass transition. For $r=0.24$ there is only one glass phase, while for $r=0.3$ and $r=0.34$ there are two glass phases. For $r=0.34$ the discontinuous glass-glass transition is disconnected from the liquid phase.

mixture with $(a, b, c)=(2,3,4)$ on a Bethe lattice with connectivity $z=5$ is particularly useful because one can get explicit analytic results. The fixed-point equation obeyed by $B$ in this case is:

$$
\frac{1}{p}=1+B-5 B^{2}+3 B^{3}+6 q B^{2}(1-B)+4 r B^{3} .
$$

Plugging $B=1$ in the fixed-point equation one get the continuous glass transition $T_{\mathrm{c}}(r)=$ $-1 / \ln (4 r-1)$. It does not depend on $q$ and is limited to the range $1 / 2 \geq r \geq 1 / 4$ (we do not consider here the case of negative temperature). Setting the first-order derivative of Eq. (6) to zero, we get

$$
q=\frac{-9 B^{2}+10 B-1-12 r B^{2}}{12 B-18 B^{2}},
$$

and thus the discontinuous transition is obtained by plotting Eqs. (6) and (7) parametrically in terms of $B$. A section of the phase diagram illustrating the distinct topologies of the transition lines obtained for different values of $r$ (the fraction of spins with $f=4$ ) is shown in the Fig. 1 along with the characteristic higher-order glass singularities. For small values of $r$ we see that there is only a discontinuous liquid-glass transition (corresponding to the fold bifurcation). The continuous transition occurs for $r>0.27$ and crosses the discontinuous line. This latter, in turn, enters the frozen phase thus producing an extra 


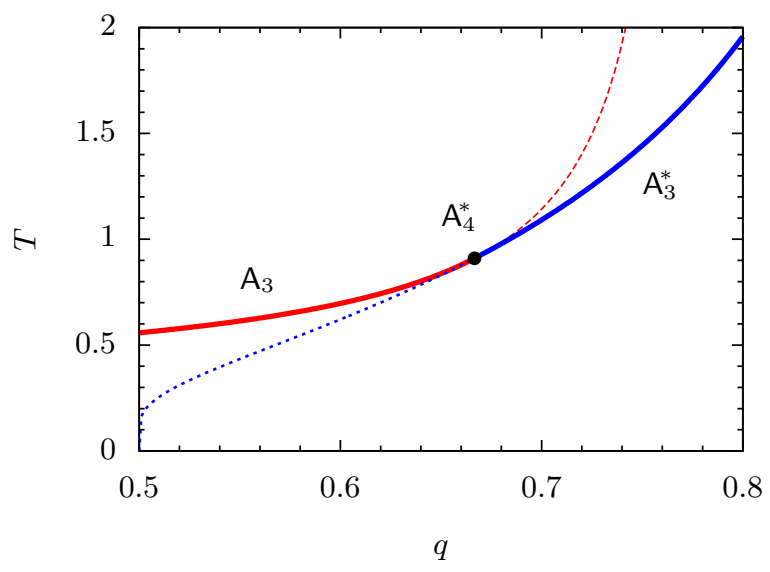

FIG. 2. Higher-order singularities in the mixture $(2,3,4)$ on a Bethe lattice with $z=5$. The transition lines corresponding to generic and degenerate cusp bifurcations are denoted with $\mathrm{A}_{3}$ and $\mathrm{A}_{3}^{*}$, respectively. They coalesce smoothly in a degenerate $\mathrm{A}_{4}^{*}$ swallowtail singularity. The dashed and dotted curves belong to the unstable branches of the transition lines.

glass-glass transition. Upon further increasing $r$ something more interesting happens: we observe that the glass-to-glass transition departs from the continuous liquid-glass transition and becomes completely disconnected from the liquid phase. This departure generates an extra endpoint singularity corresponding to a generic cusp bifurcation, $\mathrm{A}_{3}$. The range of $q$ values over which the transition is disconnected widens until the the glass-glass transition eventually disappears.

The endpoints of the glass-glass transition lines define a set of generic cusp singularities, $A_{3}$, whereas the separation points between the continuous and discontinuous liquid-glass transitions correspond to degenerate cusp singularities, $A_{3}^{*}$. They are respectively given by

$$
\frac{1}{T(q)}= \begin{cases}\ln (15-18 q), & \mathrm{A}_{3} \\ -\ln (2 q-1), & \mathrm{A}_{3}^{*}\end{cases}
$$

Either curves possess an unstable branch and are represented in the $(T, q)$ plane in Fig. 2 . One can easily check that they coaelesce smoothly in a degenerate swallowtail singularity, $\mathrm{A}_{4}^{*}$, which is exactly located at $q=2 / 3, r=1 / 3$, and $T=1 / \ln 3$. Notice that these values of $q$ and $r$ corresponds to a mixture with a perfectly balanced composition of each components $(1-q=q-r=r=1 / 3)$. For completeness we also show, in Fig. 3, the variation of the order parameter with temperature for a value of $r$ in the range in which we observe the disconnected glass-glass transition. One can easily verify that the leading behavior of both 


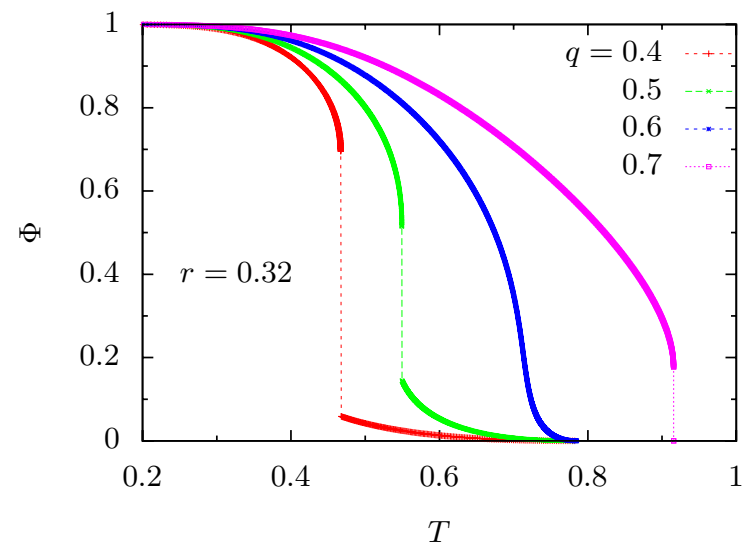

FIG. 3. The order parameter, $\Phi$, representing the fraction of frozen spins vs temperature, $T$, at $r=0.32$ and for several values of $q$ in a mixture $(2,3,4)$ with $z=5$.

$1-B$ and the order parameter $\Phi$ near the degenerate higher-order singularities $A_{3}^{*}$ and $A_{4}^{*}$ is proportional to $\epsilon^{1 / 2}$ and $\epsilon^{1 / 3}$, respectively. Whereas, near the $\mathrm{A}_{3}$ points is $1-B \sim \Phi \sim \epsilon^{1 / 3}$ ( $\epsilon$ is the variation of the control parameters).

The above findings have not been previously reported and we expect they should be present in other systems, e.g., in fluid mixtures confined in porous media and in asymmetric mixtures of hard-spheres [14]. In particular, building on the observations of Refs. [24, 25], we suggest that spin-glass models with multispin interaction terms when supplemented with an extra random field should reproduce the structure of MCT for a binary mixture in a random environment.

\section{MIXTURE $(2,5,7)$}

Next, we consider Eq. (4) with facilitation values $(a, b, c)=(2,5,7)$ on a Bethe lattice with $z=12$. This corresponds to a mixture with moderate cooperative dynamics and with a more sparse distribution of facilitation. Since there is no spin population with strongly cooperative dynamics (i.e., it is neither $f_{i}=z-1$ nor $f_{i}=z$ ) the order parameter cannot vanish continuously and so no degenerate higher-order singularity is expected in this case. A section of the phase diagram is reported in the Fig. 4: the various values of $r$, corresponding the fraction of spins with $f_{i}=7$, illustrates the different topologies of the transition lines. For $r<0.4$ we find that there is only a discontinuous liquid-glass transition, i.e., a fold bifur- 


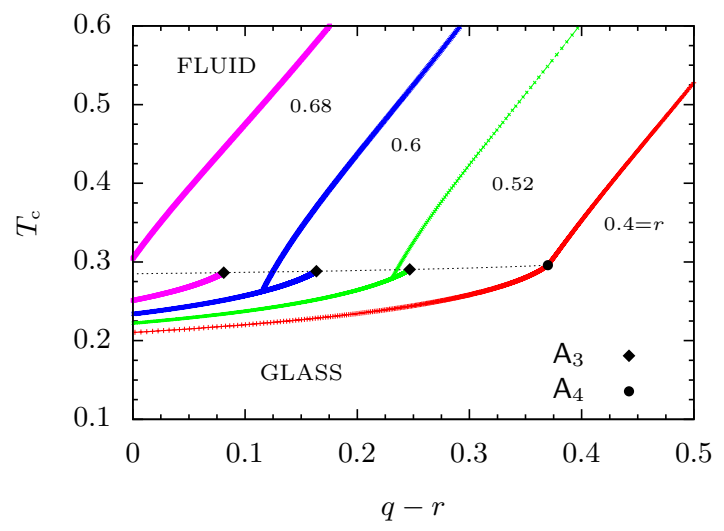

FIG. 4. A section of the phase diagram for the mixture $f=(2,5,7)$ on a Bethe lattice with connectivity $z=12$ for $r=0.4,0.52,0.6$ and 0.68 . The full lines represent discontinuous transitions while the dots correspond to higher-order singularities with cusp and swallowtail structures, respectively denoted with $\mathrm{A}_{3}$ and $\mathrm{A}_{4}$. Notice that for $r=0.68$ the glass-glass transition line is disconnected from the liquid phase.

cation, or $\mathrm{A}_{2}$ singularity. For $r>0.4$ two distinct glass states appear in the phase diagram and, correspondingly, there is a glass-glass transition line whose endpoint defines a cusp bifurcation, $A_{3}$. The line of $A_{3}$ singularities, represented by a dotted line in Fig. 4, terminates in a swallowtail bifurcation, $\mathrm{A}_{4}$, which is located at $q \simeq 0.773, T \simeq 0.296, r \simeq 0.40369$. Upon increasing further $r$ the glass-glass transition line becomes eventually disconnected from the liquid phase, see the curve $r=0.68$ in Fig. 4. Therefore, also in this case, the spin populations with different facilitation values compete with each other to produce a disconnected phase diagram. As we can observe in Fig. 4 this occurs when the fraction $1-q$ of spins with $f=2$ become smaller than that with $f=7$ (i.e., $r$ ) and, correspondingly, the fraction of spins with intermediate facilitation, $f=5$, becomes pretty small, $q \approx r$. The variation of the order parameter $\Phi$ with the temperature, $T$, is shown in Fig. 5 for $r$ in the range in which we observe the disconnected glass-glass transition and several values of $q$. As expected we find that $\Phi$ exhibits a single or a double jump depending on whether the system crosses one or two transition lines upon lowering the temperature.

The results we find here are qualitatively similar to those obtained in hard-sphere systems with the square-shoulder potential [11, 13]. Nevertheless, in the latter case, there is an interesting extra feature which is not reproduced in our approach: It is the counter-intuitive 


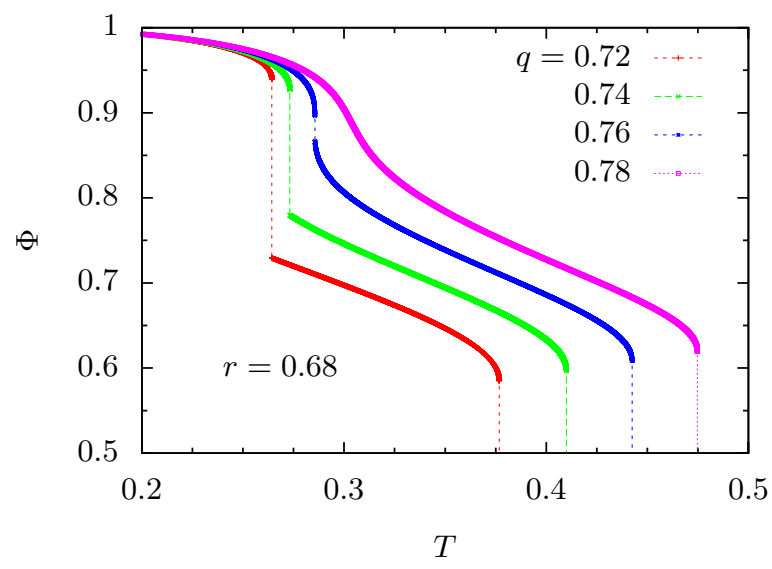

FIG. 5. The order parameter, $\Phi$, representing the fraction of frozen spins vs temperature, $T$, at $r=0.68$ and for several values of $q$, in the $(2,5,7)$ mixture with $z=12$. The double jump of $\Phi$ for $q=0.74,0.76$ and 0.78 corresponds to a discontinuous liquid-glass transition followed by a glass-glass transition upon lowering the temperature.

melting-by-cooling often associated with higher-order singularities. It would be interesting to ascertain the ingredients that are actually required to observe reentrancy in the present context, and whether they are entropic or energetic in origin.

It is also interesting to notice that the phase diagram derived above shares some similarities with that of some spin-glass models [26, 28], in particular see Fig. 1 in Ref. [26]. The disconnected glass-glass transition, however, has not been observed in such disordered systems. Since a multispin interaction term plays the same role of a facilitation dynamics with $z-2 \geq f_{i} \geq 2$, our approach suggests that an additional multispin term would be needed to observe a disconnected glass-glass transition. Nevertheless, the analysis of this latter case could be rather awkward as it involves delicate aspects of replica symmetry breaking calculations [27].

\section{CONCLUSIONS}

To summarise, I have shown that disconnected glass-glass transitions take place in systems with either a continuous or a discontinuous liquid-glass transition and that the related cusp and swallowtail bifurcations can be degenerate or generic. Such features are controlled primarily by the competition between packing effects on different microscopic length scales, 
as opposed to system-specific details of the molecular interactions. They are therefore suggested to appear in a range of soft matter systems including fluid mixtures confined in porous media, and colloidal and polymer gels. The fact that, starting from very different premises, we reach conclusions quite similar to those obtained in more realistic model systems is neither obvious nor coincidental, and should lend further support to the universality of both MCT and the present framework.

Although the dynamics of facilitated systems can be numerically simulated with a relatively modest effort (using continuous-time algorithms) one should be able to rationalise the anomalous logarithmic relaxation near higher-order singularities through the study of minimal size rearrangements [29]. Also, it would be important to identify the conditions under which the crucial parameter exponent, typically denoted with $\lambda$ in MCT, can be derived in the present context starting from the "facilitated" analogue of the MCT kernel [21]. That would provide a direct route to the determination of $\lambda$ that could be compared with the computation of multispin correlations recently suggested in Ref. [30]. Finally, it would be interesting to see whether the disconnected glass-glass transition is recovered in the replica approach to hard-sphere packings [31]. Work in these directions is in progress.

[1] W. Götze, Complex dynamics of glass-forming liquids, (Oxford University Press, Oxford, 2009).

[2] K. Dawson, G. Foffi, M. Fuchs, W. Götze, F. Sciortino, M. Sperl, P. Tartaglia, T. Voigtmann, and E. Zaccarelli, Phys. Rev. E 63, 011401 (2000).

[3] K.N. Pham, A.M. Puertas, J. Bergenholtz, S.U. Egelhaaf, A. Moussaid, P.N. Pusey, A.B. Schofield, M.E. Cates, M. Fuchs, and W.C. Poon, Science 296, 104 (2002).

[4] T. Eckert and E. Bartsch, Phys. Rev. Lett. 89, 125701 (2002).

[5] S. H. Chen, W. R. Chen, and F. Mallamace, Science 300, 619 (2003).

[6] F. Sciortino, P. Tartaglia, and E. Zaccarelli, Phys. Rev. Lett. 91, 268301 (2003).

[7] S.H. Chong, A.J. Moreno, F. Sciortino, W. Kob, Phys. Rev. Lett. 94, 215701 (2005).

[8] V. Krakoviack, Phys. Rev. Lett. 94, 065703 (2005).

[9] J. Kurzidim, D. Coslovich and G. Kahl, Phys. Rev. Lett. 103, 138303 (2009).

[10] K. Kim, K. Miyazaki, and S. Saito, Europhys. Lett. 88, 36002 (2009). 
[11] M. Sperl, E. Zaccarelli, F. Sciortino, P. Kumar, and H. E. Stanley, Phys. Rev. Lett. 104, 145701 (2010).

[12] M. Sperl, Prog. Theor. Phys. Suppl. 184, 211 (2010).

[13] G. Das, N. Gnan, F. Sciortino, and E. Zaccarelli, Unveiling the complex glassy dynamics of square shoulder systems: simulations and theory, submitted to J. Chem. Phys. (2013).

[14] Th. Voigtmann, Europhys. Lett. 96, 36006 (2011).

[15] F. Tchangnwa Nya, A. Ayadim, Ph. Germain and S. Amokrane, J. Phys. Condens. Matter 24, 325106 (2012).

[16] G.H. Fredrickson and H.C. Andersen, Phys. Rev. Lett. 53, 1244 (1984).

[17] J. Chalupa and P. L. Leath and G. R. Reich, J. Phys. C: Solid State Phys. 12, L31 (1979).

[18] N.S. Branco, J. Stat. Phys. 70, 1035 (1993).

[19] M. Sellitto, D. De Martino, F. Caccioli, and J.J. Arenzon, Phys. Rev. Lett. 105, 265704 (2010).

[20] J. J. Arenzon and M. Sellitto, J. Chem. Phys. 137, 084501 (2012).

[21] M. Sellitto, Phys. Rev. E 86, 030502(R) (2012).

[22] G.J. Baxter, S.N. Dorogovtsev, A.V. Goltsev, and J.F.F. Mendes, Phys. Rev. E 82, 011103 (2010).

[23] D. Cellai and J.P. Gleeson, in: Complex Networks IV, Studies in Computational Intelligence (Springer Berlin, 2013), vol. 476, pp. 165-172; D. Cellai, A. Lawlor, K.A. Dawson, and J.P. Gleeson, Phys. Rev. Lett. 107, 175703 (2011).

[24] S. Ciuchi and A. Crisanti, Europhys. Lett. 49, 754 (2000).

[25] V. Krakoviack, Phys. Rev. E 75, 031503 (2007).

[26] V. Krakoviack, Phys Rev. B 76, 136401 (2007).

[27] A. Crisanti and L. Leuzzi, Phys. Rev. B 76, 136402 (2007).

[28] A. Caiazzo, A. Coniglio and M. Nicodemi, Phys. Rev. Lett. 93, 215701 (2004).

[29] A. Montanari and G. Semerjian, J. Stat. Phys. 124, 103 (2006).

[30] F. Caltagirone, U. Ferrari, L. Leuzzi, G. Parisi, F. Ricci-Tersenghi, T. Rizzo, Phys. Rev. Lett. 108, 085702 (2012).

[31] G. Parisi and F. Zamponi, Rev. Mod. Phys. 82, 789 (2010). 